\title{
On metric Diophantine approximation in positive characteristic
}

by

Kae Inoue and Hitoshi Nakada (Yokohama)

1. Introduction. Let $\mathbb{F}$ be a finite field with $q$ elements. We denote by $\mathbb{F}[X]$ the set of polynomials with coefficients in $\mathbb{F}$, and by $\mathbb{F}(X)$ the quotient field of $\mathbb{F}[X]$. We also denote by $\mathbb{F}\left(\left(X^{-1}\right)\right)$ the set of formal Laurent power series:

$$
\mathbb{F}\left(\left(X^{-1}\right)\right)=\left\{f=a_{l} X^{l}+a_{l-1} X^{l-1}+\ldots: l \in \mathbb{Z} \text { and each } a_{i} \in \mathbb{F}\right\} .
$$

For $f \in \mathbb{F}\left(\left(X^{-1}\right)\right)$, we denote by $[f]$ its polynomial part:

$$
[f]=a_{l} X^{l}+a_{l-1} X^{l-1}+\ldots+a_{0},
$$

and define

$$
|f|= \begin{cases}0 & \text { if } f=0, \\ q^{l} & \text { if } a_{l} \neq 0 .\end{cases}
$$

In this paper, we discuss the metric theory of Diophantine approximation of Laurent series on the analogy of the classical theory; here, $\mathbb{F}[X], \mathbb{F}(X)$, and $\mathbb{F}\left(\left(X^{-1}\right)\right)$ play the role of integers, rational numbers, and real numbers, respectively. We put

$$
\mathbb{L}=\left\{f=a_{-1} X^{-1}+a_{-2} X^{-2}+\ldots: a_{i} \in \mathbb{F} \text { for } i \leq-1\right\},
$$

which plays the role of the unit interval $[0,1)$. Then $\mathbb{L}$ is a compact Abelian group with the metric $d(f, g)=|f-g|$. We denote by $m$ the normalized Haar measure on $\mathbb{L}$. Note that

$$
m\left\{f=a_{-1} X^{-1}+a_{-2} X^{-2}+\ldots: a_{-1}=b_{1}, \ldots, a_{-l}=b_{l}\right\}=\frac{1}{q^{l}}
$$

for any $b_{1}, \ldots, b_{l} \in \mathbb{F}$. Our aim is to study the following Diophantine inequality:

$$
\left|f-\frac{P}{Q}\right|<\frac{\psi(Q)}{|Q|}, \quad P, Q \text { coprime, }
$$

where $\psi$ is a non-negative function defined on $\mathbb{F}[X]$ and $\psi(Q)=\psi\left(Q^{\prime}\right)$ whenever $Q^{\prime}=a Q$ for some non-zero $a \in \mathbb{F}$. The question is whether this

2000 Mathematics Subject Classification: 11J61, 11K60. 
inequality has an infinite number of solutions $P / Q$ for $m$-a.e. $f \in \mathbb{L}$. In the case of real numbers:

$$
\left|x-\frac{p}{q}\right|<\frac{\psi(q)}{q}, \quad(p, q)=1,
$$

a number of sufficient conditions are known for this question. For example, if $q \psi(q)$ is non-increasing, then (1) has infinitely many solutions for a.e. $x \in \mathbb{R}$ if and only if $\sum \psi(q)$ diverges. This can be proved by using the continued fraction expansion of $x$ (see Billingsley [2], for example). We refer to [7] and [5] for the formal power series version of this theorem. In general, we cannot make use of continued fractions for this type of problem. We refer to [9] for general cases. In what follows, we first restrict to the case where $\psi(Q)$ depends only on the degree of $Q$. In this case, it is easy to give a necessary and sufficient condition on $\psi$ for having infinitely many solutions for a.e. $f \in \mathbb{L}$. Indeed, we have the following:

THEOREM 1. Let $\psi$ be a non-negative function defined on $\mathbb{F}[X]$ such that $\psi(Q)$ depends only on the degree of $Q \in \mathbb{F}[X]$. For any set $S$ of positive integers, the inequality

$$
\left|f-\frac{P}{Q}\right|<\frac{\psi(Q)}{|Q|}
$$

with $P, Q$ coprime and $\operatorname{deg} Q \in S$ has infinitely many solutions for almost every $f \in \mathbb{L}$ if and only if

$$
\sum_{d \in S} q^{d} \psi\left(X^{d}\right)=\infty
$$

To prove this theorem, we use continued fractions over $\mathbb{F}(X)$ to compute the number of fractions $P / Q$ with $\operatorname{deg} Q=n$ for $n \geq 1$ (see [3]). We discuss this in Section 2 and give the proof in Section 3. If $\psi(Q)$ does not depend only on the degree of $Q$, then it is not easy to give a necessary and sufficient condition for the existence of infinitely many solutions (a.e.). Let $\psi$ be a $\left\{q^{-n}: n \geq 0\right\} \cup\{0\}$-valued function defined on the set of monic polynomials in $\mathbb{F}[X]$ of the form

$$
X^{l}+a_{l-1} X^{l-1}+\ldots+a_{1} X+a_{0}, \quad a_{i} \in \mathbb{F}, 0 \leq i \leq l-1 .
$$

We denote by $E$ the set of $f \in \mathbb{L}$ such that the inequality

$$
\left|f-\frac{P}{Q}\right|<\frac{\psi(Q)}{|Q|}, \quad P, Q \text { coprime, } Q \text { monic, }
$$

has infinitely many solutions. In Section 4 , we prove that $m(E)=0$ or 1 (Theorem 4), which is an analogue of Gallagher's theorem (see [6]). Moreover we show that the Duffin-Schaeffer type theorem (see [4] and [9] for the classical case) holds. 
THEOREM 2. Let $\psi$ be $a\left\{q^{-n}: n \geq 0\right\} \cup\{0\}$-valued function which satisfies

$$
\sum_{n=1}^{\infty} \sum_{\substack{\operatorname{deg} Q=n \\ Q \text { monic }}} \psi(Q)=\infty .
$$

Suppose there are infinitely many positive integers $n$ such that

$$
\sum_{\substack{\operatorname{deg} Q \leq n \\ Q \text { monic }}} \psi(Q)<C \sum_{\substack{\operatorname{deg} Q \leq n \\ Q \text { monic }}} \psi(Q) \frac{\Phi(Q)}{|Q|}
$$

for a constant $C$. Then the inequality

$$
\left|f-\frac{P}{Q}\right|<\frac{\psi(Q)}{|Q|}, \quad(P, Q)=1,
$$

has infinitely many solutions $P / Q$ for a.e. $f \in \mathbb{L}$.

Here, $(P, Q)=1$ means that $P$ and $Q$ are coprime polynomials, and $\Phi(Q)$ is the number of monic polynomials $Q^{\prime}$ such that

$$
\operatorname{deg} Q^{\prime}<\operatorname{deg} Q, \quad\left(Q, Q^{\prime}\right)=1 .
$$

2. Continued fractions. We refer to Berthé and Nakada [1] for the details of the continued fraction expansions of power series.

Let $T$ be the map of $\mathbb{L}$ onto itself defined by

$$
T(f)=f^{-1}-\left[f^{-1}\right], \quad f \in \mathbb{L} .
$$

Henceforth, we denote by 1 the unity of multiplication of $\mathbb{F}$, and by 0 the unity of addition. Then we have

$$
f=\frac{1}{p_{1}+\frac{1}{p_{2}+\ddots}}=:\left[0 ; p_{1}, p_{2}, \ldots\right] \text { with } \quad p_{n}=\left[\left(T^{n-1} f\right)^{-1}\right] .
$$

As in the classical case, we define

$$
\begin{aligned}
& P_{n}=p_{n} P_{n-1}+P_{n-2}, \quad P_{0}=0, \quad P_{1}=1, \\
& Q_{n}=p_{n} Q_{n-1}+Q_{n-2}, \quad Q_{0}=1, \quad Q_{1}=p_{1} \text {, }
\end{aligned}
$$

and we have

$$
\begin{aligned}
& P_{n} Q_{n-1}-Q_{n} P_{n-1}= \pm 1, \\
& \frac{P_{n}}{Q_{n}}=\frac{1}{p_{1}+\frac{1}{p_{2}+\ddots \cdot+\frac{1}{p_{n}}}}=:\left[0 ; p_{1}, \ldots, p_{n}\right] \quad \text { for } n \geq 1 \text {. }
\end{aligned}
$$


We call $P_{n} / Q_{n}$ the $n$th convergent fraction of $f$. Since

$$
f=\frac{P_{n}+T^{n} f \cdot P_{n-1}}{Q_{n}+T^{n} f \cdot Q_{n-1}}
$$

it is easy to see that

$$
\left|f-\frac{P_{n}}{Q_{n}}\right|<\frac{1}{\left|Q_{n}\right|^{2}} \quad \text { for } n \geq 1 .
$$

Moreover, we have the following:

Lemma 1. If two relatively prime non-zero polynomials $P, Q$ satisfy

$$
\left|f-\frac{P}{Q}\right|<\frac{1}{|Q|^{2}}
$$

then

$$
\frac{P}{Q}=\frac{P_{n}}{Q_{n}} \quad \text { for some } n \geq 1
$$

We put

$$
W_{n}=\left\{\frac{P}{Q} \in \mathbb{L}: \operatorname{deg} Q=n,(P, Q)=1\right\} \quad \text { for } n \geq 1 .
$$

The following lemma, shown in [3], is essential in the next section. Here we prove it by the use of continued fractions.

Lemma 2.

$$
\# W_{n}=q^{2 n}-q^{2 n-1} \quad \text { for } n \geq 1 .
$$

Proof. If $n=1$, all elements in $W_{1}$ are of the form

$$
\frac{P}{Q}=\frac{a}{X+b} \quad \text { with } a, b \in \mathbb{F}, a \neq 0 .
$$

This implies the assertion. Now suppose

$$
\# W_{i}=q^{2 i}-q^{2 i-1} \quad \text { for } 1 \leq i \leq n .
$$

Fix $P / Q \in W_{n+1}$. Then we have a unique continued fraction expansion

$$
\frac{P}{Q}=\left[0 ; p_{1}, \ldots, p_{m}\right]
$$

So we can define a unique element $P^{\prime} / Q^{\prime} \in W_{j}$ for some $j, 1 \leq j \leq n$, by

$$
\frac{P^{\prime}}{Q^{\prime}}=\left[0 ; p_{1}, \ldots, p_{m-1}\right]
$$

unless $m=1$. On the other hand, for any $P^{\prime} / Q^{\prime} \in W_{j}, 1 \leq j \leq n$, we have $q^{n+1-j}(q-1)$ fractions $P / Q \in W_{n+1}$ by (4). The number of $P / Q$ with 
$\operatorname{deg} Q=n+1$ and $\operatorname{deg} P=0$ is $q^{n+1}(q-1)$. Thus

$$
\begin{aligned}
\# W_{n+1} & =\sum_{j=1}^{n} q^{n+1-j}(q-1)\left(q^{2 j}-q^{2 j-1}\right)+q^{n+1}(q-1) \\
& =q^{2 n+2}-q^{2 n+1}
\end{aligned}
$$

3. Proof of Theorem 1. In what follows, we always assume that $P$ and $Q$ are coprime non-zero polynomials whenever $P / Q$ denotes a rational function.

For $P / Q$ with $\operatorname{deg} Q=n$, we put

$$
E_{n}\left(\frac{P}{Q}\right)=\left\{f \in \mathbb{L}:\left|f-\frac{P}{Q}\right|<\frac{1}{q^{2 n}}\right\}
$$

and also put

$$
E_{n}=\left\{f \in \mathbb{L}: \text { there exists } \frac{P}{Q} \text { such that } \operatorname{deg} Q=n,\left|f-\frac{P}{Q}\right|<\frac{1}{q^{2 n}}\right\} .
$$

Lemma 3. For a fixed integer $n \geq 1$, if $P / Q \neq P^{\prime} / Q^{\prime}$ with $\operatorname{deg} Q=$ $\operatorname{deg} Q^{\prime}=n$, then

$$
E_{n}\left(\frac{P}{Q}\right) \cap E_{n}\left(\frac{P^{\prime}}{Q^{\prime}}\right)=\emptyset .
$$

Proof. Since $|\cdot|$ is ultrametric, we see that if the intersection were nonempty, then

$$
\left|\frac{P}{Q}-\frac{P^{\prime}}{Q^{\prime}}\right|<\frac{1}{q^{2 n}}
$$

However,

$$
\left|\frac{P}{Q}-\frac{P^{\prime}}{Q^{\prime}}\right| \geq \frac{1}{|Q|\left|Q^{\prime}\right|}=\frac{1}{q^{2 n}},
$$

which gives a contradiction.

Lemma 4. For any $n \geq 1$,

$$
m\left(E_{n}\right)=1-\frac{1}{q} .
$$

Proof. Since $m\left\{f \in \mathbb{L}:|f-P / Q|<1 / q^{2 n}\right\}=1 / q^{2 n}$ for a fixed $P / Q$ with $\operatorname{deg} Q=n$, and the number of $P / Q$ is $q^{2 n}-q^{2 n-1}$ from Lemma 2, we have the assertion.

LEMma 5. For any $n \geq 1$ and $k \geq 1$, we have

$$
m\left(E_{n} \cap E_{n+k}\right)=m\left(E_{n}\right) m\left(E_{n+k}\right)=\left(1-\frac{1}{q}\right)^{2} .
$$


Proof. If $f \in E_{n} \cap E_{n+k}$, say

$$
\left|f-\frac{P}{Q}\right|<\frac{1}{q^{2 n}}, \quad\left|f-\frac{P^{\prime}}{Q^{\prime}}\right|<\frac{1}{q^{2 n+2 k}}
$$

with $\operatorname{deg} Q=n, \operatorname{deg} Q^{\prime}=n+k$, then $\left|P^{\prime} / Q^{\prime}-P / Q\right|<1 / q^{2 n}$, so that by Lemma $1, P / Q$ is a convergent of the continued fraction of $P^{\prime} / Q^{\prime}$. Conversely, when this is the case, and $\left|f-P^{\prime} / Q^{\prime}\right|<1 / q^{2 n+2 k}$, then $f \in$ $E_{n} \cap E_{n+k}$. Therefore

$$
m\left(E_{n} \cap E_{n+k}\right)=Z(n, n+k) \frac{1}{q^{2 n+2 k}},
$$

where $Z(n, n+k)$ is the number of pairs $P / Q, P^{\prime} / Q^{\prime}$ with $P / Q$ a convergent to $P^{\prime} / Q^{\prime}$, and $\operatorname{deg} Q=n, \operatorname{deg} Q^{\prime}=n+k$. The number of choices for $P / Q$ is $\# W_{n}=q^{2 n}(1-1 / q)$. For given $P / Q$, we will find the number of choices for $P^{\prime} / Q^{\prime}$. Suppose that $P^{\prime} / Q^{\prime}$ satisfies

$$
\left|f-\frac{P^{\prime}}{Q^{\prime}}\right|<\frac{1}{q^{2 n+2 k}}, \quad \operatorname{deg} Q^{\prime}=n+k \quad \text { for } f \in E_{n}\left(\frac{P}{Q}\right) .
$$

There exist $n=j_{0}<j_{1}<\ldots<j_{l-1}<j_{l}=n+k$ (uniquely) such that

$$
\frac{P^{\prime}}{Q^{\prime}}=\frac{P_{m+l}}{Q_{m+l}}=\left[0 ; p_{1}, p_{2}, \ldots, p_{m}, \ldots, p_{m+l}\right]
$$

with

$$
\operatorname{deg} p_{m+i}=j_{i}-j_{i-1}, \quad 1 \leq i \leq l .
$$

Since $\#\{p \in \mathbb{F}[X]: \operatorname{deg} p=u\}=q^{u}(q-1)$, we have

$$
\begin{aligned}
\#\left\{\frac{P^{\prime}}{Q^{\prime}}: \operatorname{deg} p_{m+i}=j_{i}-j_{i-1}, 1 \leq i \leq l\right\} \\
\quad=q^{j_{1}-j_{0}}(q-1) q^{j_{2}-j_{1}}(q-1) \ldots q^{j_{l}-j_{l-1}}(q-1)=q^{k}(q-1)^{l}
\end{aligned}
$$

for each fixed $\left(j_{1}, \ldots, j_{l}\right)$. The number of choices for $n<j_{1}<\ldots<j_{l-1}$ $<n+k$ is $\left(\begin{array}{c}k-1 \\ l-1\end{array}\right)$ and $l$ runs from 1 to $k$. Hence

$$
\begin{aligned}
\#\left\{\frac{P^{\prime}}{Q^{\prime}}:\left|f-\frac{P^{\prime}}{Q^{\prime}}\right|<\frac{1}{q^{2 n+2 k}} \text { for some } f \in E_{n}\left(\frac{P}{Q}\right)\right\} \\
\quad=\sum_{l=1}^{k}\left(\begin{array}{c}
k-1 \\
l-1
\end{array}\right) q^{k}(q-1)^{l}=q^{2 k}\left(1-\frac{1}{q}\right) .
\end{aligned}
$$

Consequently,

$$
Z(n, n+k)=q^{2 n+2 k}\left(1-\frac{1}{q}\right)^{2}
$$

and by (5), we get

$$
m\left(E_{n} \cap E_{n+k}\right)=\left(1-\frac{1}{q}\right)^{2}=m\left(E_{n}\right) m\left(E_{n+k}\right) .
$$


By the Borel-Cantelli lemma, this implies the following:

Proposition 1. For any sequence $n_{1}<n_{2}<\ldots$ of positive integers the inequality

$$
\left|f-\frac{P}{Q}\right|<\frac{1}{|Q|^{2}}, \quad \operatorname{deg} Q=n_{i},
$$

has infinitely many solutions for $m$-a.e. $f \in \mathbb{L}$.

According to this proposition, we can assume that $\psi(Q)<1 / q^{n}$ for any $n \geq 1$. Then we rewrite Theorem 1 as follows:

TheOREM 3. For any sequences $n_{1}<n_{2}<\ldots$ and $l_{1}, l_{2}, \ldots$ of positive integers, the inequality

$$
\left|f-\frac{P}{Q}\right|<\frac{1}{q^{2 n_{i}+l_{i}}}, \quad \operatorname{deg} Q=n_{i},
$$

has infinitely many solutions for $m$-a.e. $f \in \mathbb{L}$ if and only if

$$
\sum_{i=1}^{\infty} q^{-l_{i}}=\infty .
$$

Proof. Put

$$
F_{i}=\left\{f \in \mathbb{L}: \text { there exists } \frac{P}{Q} \text { such that }\left|f-\frac{P}{Q}\right|<\frac{1}{q^{2 n_{i}+l_{i}}}, \operatorname{deg} Q=n_{i}\right\} .
$$

Given $P / Q$, the measure of $f \in \mathbb{L}$ with $|f-P / Q|<1 / q^{2 n_{i}+l_{i}}$ is $1 / q^{2 n_{i}+l_{i}}$. The number of $P / Q$ in $W_{n_{i}}$ is $q^{2 n_{i}}-q^{2 n_{i}-1}$, therefore

$$
m\left(F_{i}\right)=\frac{q-1}{q} \cdot \frac{1}{q^{l_{i}}} .
$$

Now the assertion follows from the next lemma together with (6) by Theorem 3 of [8].

Lemma 6. (a) $F_{i} \cap F_{i+j}=\emptyset$ if $n_{i}+l_{i} \geq n_{i+j}$.

(b) $m\left(F_{i} \cap F_{i+j}\right)=m\left(F_{i}\right) m\left(F_{i+j}\right)$ if $n_{i}+l_{i}<n_{i+j}$.

Proof. If $f \in F_{i} \cap F_{i+j}$, say

$$
\left|f-\frac{P}{Q}\right|<\frac{1}{q^{2 n_{i}+l_{i}}}, \quad\left|f-\frac{P^{\prime}}{Q^{\prime}}\right|<\frac{1}{q^{2 n_{i+j}+l_{i+j}}}
$$

with $\operatorname{deg} Q=n_{i}, \operatorname{deg} Q^{\prime}=n_{i+j}$, then

$$
\left|\frac{P}{Q}-\frac{P^{\prime}}{Q^{\prime}}\right|<\frac{1}{q^{2 n_{i}+l_{i}}},
$$

and on the other hand

$$
\left|\frac{P}{Q}-\frac{P^{\prime}}{Q^{\prime}}\right| \geq \frac{1}{|Q|\left|Q^{\prime}\right|}=\frac{1}{q^{n_{i}+n_{i+j}}} .
$$

When $n_{i}+l_{i} \geq n_{i+j}$ these inequalities contradict each other, so $F_{i} \cap F_{i+j}=\emptyset$. 
Suppose, then, that $n_{i}+l_{i}<n_{i+j}$. It follows from (7) that $P / Q$ is a convergent to $P^{\prime} / Q^{\prime}$. Write again

$$
\frac{P}{Q}=\left[0 ; p_{1}, \ldots, p_{m}\right], \quad \frac{P^{\prime}}{Q^{\prime}}=\left[0 ; p_{1}, \ldots, p_{m}, p_{m+1}, \ldots, p_{m+l}\right] .
$$

Then by a well-known formula,

$$
\left|\frac{P}{Q}-\frac{P^{\prime}}{Q^{\prime}}\right|=\frac{1}{|Q|^{2}\left|p_{m+1}\right|}=\frac{1}{q^{2 n_{i}+\operatorname{deg} p_{m+1}}},
$$

yielding $\operatorname{deg} p_{m+1}>l_{i}$. In analogy to (5) we obtain

$$
m\left(F_{i} \cap F_{i+j}\right)=Z\left(n_{i}, n_{i+j}, l_{i}\right) \frac{1}{q^{2 n_{i+j}+l_{i+j}}},
$$

where $Z\left(n_{i}, n_{i+j}, l_{i}\right)$ is the number of pairs $P / Q, P^{\prime} / Q^{\prime}$ as above with $\operatorname{deg} p_{m+1}>l_{i}$. Now, the number of choices for $p_{m+1}, \ldots, p_{m+l}$ is

$$
q^{\operatorname{deg} p_{m+1}}(q-1) q^{\operatorname{deg} p_{m+2}}(q-1) \ldots q^{\operatorname{deg} p_{m+l}}(q-1)=q^{n_{i+j}-n_{i}}(q-1)^{l} .
$$

Thus

$$
\begin{aligned}
& Z\left(n_{i}, n_{i+j}, l_{i}\right) \\
& \quad=\left(q^{2 n_{i}}-q^{2 n_{i}-1}\right) \sum_{l=1}^{n_{i+j}-n_{i}-l_{i}}\left(\begin{array}{c}
n_{i+j}-n_{i}-l_{i}-1 \\
l-1
\end{array}\right) q^{n_{i+j}-n_{i}}(q-1)^{l} \\
& \quad=\left(q^{2 n_{i}}-q^{2 n_{i}-1}\right) q^{n_{i+j}-n_{i}}(q-1) q^{n_{i+j}-n_{i}-l_{i}-1} \\
& \quad=q^{2 n_{i+j}-l_{i}}\left(1-\frac{1}{q}\right)^{2}
\end{aligned}
$$

which together with (8) yields the lemma.

Example 1. Put

$$
\psi(Q)= \begin{cases}1 /|Q| & \text { if } \operatorname{deg} Q \text { is prime } \\ 0 & \text { otherwise }\end{cases}
$$

Then we see that there are infinitely many solutions of

$$
\left|f-\frac{P}{Q}\right|<\frac{1}{|Q|^{2}}, \quad \operatorname{deg} Q \text { prime, }
$$

for a.e. $f \in \mathbb{L}$.

4. General case. For a given polynomial

$$
h=a_{l} X^{l}+a_{l-1} X^{l-1}+\ldots+a_{1} X+a_{0}, \quad a_{i} \in \mathbb{F}, 0 \leq i \leq l, a_{l} \neq 0,
$$

we denote by $\langle h\rangle$ the cylinder set defined by

$$
\left\{f \in \mathbb{L}:\left[X^{l+1} \cdot f\right]=h\right\} .
$$


LEMMA 7. Let $h_{k}, k \geq 1$, be a sequence of polynomials with

$$
\lim _{k \rightarrow \infty} \operatorname{deg} h_{k}=\infty,
$$

and $E_{k}$ be a sequence of measurable subsets of $\mathbb{L}$ for which $E_{k} \subset\left\langle h_{k}\right\rangle$. Suppose that $m\left(E_{k}\right) \geq \delta m\left(\left\langle h_{k}\right\rangle\right)$ for some $\delta>0$. Then

$$
m\left(\bigcap_{l=1}^{\infty} \bigcup_{k=l}^{\infty} E_{k}\right)=m\left(\bigcap_{l=1}^{\infty} \bigcup_{k=l}^{\infty}\left\langle h_{k}\right\rangle\right) .
$$

Proof. Let

$$
H:=\bigcap_{l=1}^{\infty} \bigcup_{k=l}^{\infty}\left\langle h_{k}\right\rangle, \quad E_{l}^{*}=\bigcup_{k=l}^{\infty} E_{k}, \quad H_{l}^{*}:=H \backslash E_{l}^{*} .
$$

We show that $m\left(H_{l}^{*}\right)=0$ for any $l \geq 1$, which implies the assertion of this lemma. Suppose that $m\left(H_{k}^{*}\right)>0$. For almost all $f_{0} \in H_{l}^{*}$, there are infinitely many $k$ such that $f_{0} \in\left\langle h_{k}\right\rangle$. For $f=\sum_{i<0} a_{i} X^{i} \in \mathbb{L}$, we put $\iota(f)=\sum_{i<0} a_{i} q^{i} \in(0,1]$. The map $\iota$ is a measure isomorphism of $(\mathbb{L}, m)$ to $(0,1]$ with the Lebesgue measure. By this isomorphism, the cylinder sets $\left\langle h_{k}\right\rangle$ are mapped to $q$-adic rational intervals. So we can apply Lebesgue's density theorem to get

$$
\frac{m\left(H_{k}^{*} \cap\left\langle h_{k}\right\rangle\right)}{m\left(\left\langle h_{k}\right\rangle\right)}>1-\frac{\delta}{2}
$$

for some $k$. On the other hand, $H_{k}^{*} \cap E_{k}^{*}=\emptyset$. So

$$
m\left(\left\langle h_{k}\right\rangle\right) \geq m\left(E_{k}\right)+m\left(H_{k}^{*} \cap\left\langle h_{k}\right\rangle\right) \geq \delta m\left(\left\langle h_{k}\right\rangle\right)+m\left(H_{k}^{*} \cap\left\langle h_{k}\right\rangle\right),
$$

which says that $m\left(H_{k}^{*} \cap\left\langle h_{k}\right\rangle\right) \leq(1-\delta) m\left(\left\langle h_{k}\right\rangle\right)$. This is impossible.

LEMMA 8. For any polynomial $h \in \mathbb{F}[X]$ and $g \in \mathbb{L}$, the map $T$ of $\mathbb{L}$ onto itself defined by

$$
T(f)=h f+g-[h f+g] \quad \text { for } f \in \mathbb{L}
$$

is ergodic.

Proof. It is easy to see that both $f \mapsto h \cdot f$ and $f \mapsto f+g$ for $f \in \mathbb{L}$ are $m$-preserving. Then $\omega_{i}(f)=\left[h \cdot T^{i-1}\right], 1 \leq i<\infty$, is an independent and identically distributed sequence of random variables defined on $(\mathbb{L}, m)$. This implies the assertion of the lemma.

Let $\psi$ be a $\left\{q^{-n}: n \geq 0\right\} \cup\{0\}$-valued function defined on the set of monic polynomials, that is, of the form

$$
X^{l}+a_{l-1} X^{l-1}+\ldots+a_{1} X+a_{0}, \quad a_{i} \in \mathbb{F}, 0 \leq i \leq l-1 .
$$

Here $\psi(Q)$ depends on $Q$ itself, and we put 


$$
\begin{aligned}
E_{Q}=\{f \in \mathbb{L}:|f-P / Q|<\psi(Q) /|Q| & \text { for some polynomial } P \\
& \text { with } \operatorname{deg} P<\operatorname{deg} Q \text { and }(P, Q)=1\}
\end{aligned}
$$

for a monic polynomial $Q$. The following theorem is a formal power series version of $[6]$.

TheOrem 4. For any $\psi, m\left(\bigcap_{n=1}^{\infty} \bigcup_{\operatorname{deg} Q \geq n} E_{Q}\right)=0$ or 1.

Proof. If

$$
\limsup _{\operatorname{deg} Q \rightarrow \infty} \frac{\psi(Q)}{q^{\operatorname{deg} Q}}>0
$$

then we can find a sequence $Q_{1}, Q_{2}, \ldots$ of monic polynomials and a positive integer $l$ such that $\psi\left(Q_{k}\right) / q^{\operatorname{deg} Q}>q^{-l}$ for any $k \geq 1$. For any $f \in \mathbb{L}$ and sufficiently large $k$, we can find $P\left(\operatorname{deg} P<\operatorname{deg} Q_{k}\right)$ such that

$$
\left|f-\frac{P}{Q_{k}}\right|<\frac{1}{q^{l}} \quad\left(<\frac{\psi\left(Q_{k}\right)}{q^{\operatorname{deg} Q_{k}}}\right)
$$

and $P$ and $Q_{k}$ are coprime. Otherwise, $Q_{k}$ has more than $q^{\operatorname{deg} Q_{k}-l}$ factors, which is impossible. This implies

$$
m\left(\bigcap_{l=1}^{\infty} \bigcup_{k=l}^{\infty} E_{Q_{k}}\right)=1 .
$$

Now we show the assertion of the theorem when

$$
\limsup _{\operatorname{deg} Q \rightarrow \infty} \frac{\psi(Q)}{q^{\operatorname{deg} Q}}=0
$$

This means we can apply Lemma 7 . We put

$$
E=\bigcap_{n=1}^{\infty} \bigcup_{\operatorname{deg} Q \geq n} E_{Q} .
$$

Let $R$ be an irreducible polynomial and consider

$$
\left|f-\frac{P}{Q}\right|<\frac{\psi(Q)|R|^{n-1}}{|Q|}, \quad(P, Q)=1,
$$

for $n \geq 1$. We put

$$
\begin{aligned}
& E_{0}(n: R)=\{f \in \mathbb{L}:(9) \text { has infinitely many solutions } P, Q \text { with } R \nmid Q\}, \\
& E_{1}(n: R)=\{f \in \mathbb{L}:(9) \text { has infinitely many solutions } P, Q \text { with } R \| Q\} .
\end{aligned}
$$

Then

$$
E_{i}(1: R) \subset E_{i}(2: R) \subset \ldots, \quad E_{i}(1: R) \subset E \quad \text { for } i=0,1 .
$$


From Lemma 7 , we find that $m\left(E_{i}(n: R)\right)=m\left(E_{i}(1: R)\right)$ for $n \geq 1$. Thus

$$
m\left(\bigcup_{n \geq 1} E_{i}(n: R)\right)=m\left(E_{i}(1: R)\right) .
$$

Let

$$
T_{1}(f)=R \cdot f-[R \cdot f] \quad \text { for } f \in \mathbb{L} .
$$

Then

$$
T_{1}\left(\bigcup_{n \geq 1} E_{0}(n: R)\right)=\bigcup_{n \geq 2} E_{0}(n: R) .
$$

From Lemma 8, we have

$$
m\left(\bigcup_{n \geq 1} E_{0}(n: R)\right)=0 \text { or } 1 .
$$

Next we let

$$
T_{2}(f)=R \cdot f+\frac{1}{R}-\left[R \cdot f+\frac{1}{R}\right] \quad \text { for } f \in \mathbb{L} .
$$

Suppose (9) holds. We have

$$
\left|\left(R \cdot f+\frac{1}{R}\right)-\frac{R \cdot P+Q / R}{Q}\right|<\frac{\psi(Q)|R|^{n}}{|Q|}, \quad\left(R \cdot P+\frac{Q}{R}, Q\right)=1,
$$

and so

$$
T_{2}\left(\bigcup_{n \geq 1} E_{1}(n: R)\right)=\bigcup_{n \geq 2} E_{1}(n: R) .
$$

Thus we have, again by Lemma 8 ,

$$
m\left(\bigcup_{n \geq 1} E_{1}(n: R)\right)=0 \text { or } 1 .
$$

Hence, if either $m\left(E_{0}(1: R)\right)$ or $m\left(E_{1}(1: R)\right)$ is positive for some irreducible polynomial $R$, then $m(E)=1$. Assume that $m\left(E_{0}(1: R)\right)=$ $m\left(E_{1}(1: R)\right)=0$ for any irreducible polynomial $R$. We put

$$
F(R)=\left\{f \in \mathbb{L}:(2) \text { has infinitely many solutions } P, Q \text { with } R^{2} \mid Q\right\} .
$$

If $f \in F(R)$, then

$$
\left|\left(f+\frac{U}{R}\right)-\frac{P+Q U / R}{Q}\right|<\frac{\psi(Q)}{|Q|}, \quad\left(P+\frac{Q U}{R}, Q\right)=1,
$$

for any polynomial $U$ with $0 \leq \operatorname{deg} U<\operatorname{deg} R$. This means that $f \in F(R)$ implies $f+U / R \in F(R)$. If we put $S(U ; R)=\{f \in \mathbb{L}:[R f]=U\}$, then

$$
\bigcup_{U: 0 \leq \operatorname{deg} U \leq \operatorname{deg} R} S(U ; R) \cup\{f \in \mathbb{L}: \operatorname{deg} f<-\operatorname{deg} R\}=\mathbb{L}
$$


and each measure is equal to $1 / q^{\operatorname{deg} R}$. Since $F(R)$ is $(\cdot+U / R)$-invariant,

$$
m(F(R) \cap S(U ; R))=\frac{m(F(R))}{q^{\operatorname{deg} R}} .
$$

This implies

$$
\frac{m(F(R) \cap S(U ; R))}{m(S(U ; R))}=m(F(R)) .
$$

By the density theorem, we have $m(E)=m(F(R))=1$ whenever $m(F(R))$ $>0$ for some irreducible polynomial $R$; otherwise $m(E)=0$, since $E=$ $F(R) \cup E_{0}(1, R) \cup E_{1}(1, R)$. This concludes the proof of the theorem.

Remark. Note that the set $E$ is the same as the one in the introduction.

Proof of Theorem 2. In what follows, we always assume that $Q, Q_{1}, Q^{\prime}$ and $Q_{1}^{\prime}$ are monic. By the definition of $E_{Q}$,

$$
m\left(E_{Q}\right)=\psi(Q) \frac{\Phi(Q)}{|Q|} .
$$

Now consider the measure of the intersection of $E_{Q_{1}}$ and $E_{Q}\left(\operatorname{deg} Q_{1} \leq\right.$ $\operatorname{deg} Q)$. We let $N\left(Q_{1}, Q\right)$ be the number of pairs of polynomials $P$ and $P_{1}$. For these polynomials, the conditions

$$
\begin{gathered}
\left|\frac{P}{Q}-\frac{P_{1}}{Q_{1}}\right|<\frac{\psi(Q)}{|Q|}+\frac{\psi\left(Q_{1}\right)}{\left|Q_{1}\right|}, \\
(P, Q)=\left(P_{1}, Q_{1}\right)=1, \quad \operatorname{deg} P<\operatorname{deg} Q, \quad \operatorname{deg} P_{1}<\operatorname{deg} Q_{1},
\end{gathered}
$$

hold for given $Q$ and $Q_{1}$. Then

$$
m\left(E_{Q_{1}} \cap E_{Q}\right) \leq \min \left(\frac{\psi\left(Q_{1}\right)}{\left|Q_{1}\right|}, \frac{\psi(Q)}{|Q|}\right) N\left(Q_{1}, Q\right) .
$$

If

$$
P Q_{1}-P_{1} Q=R
$$

for some polynomial $R$, then $D=\left(Q_{1}, Q\right)$ divides $R$. Setting $Q_{1}=D Q_{1}^{\prime}, Q=$ $D Q^{\prime}, R=D R^{\prime}$, we have

$$
P Q_{1}^{\prime}-P_{1} Q^{\prime}=R^{\prime}, \quad\left(Q_{1}^{\prime}, Q^{\prime}\right)=1 .
$$

If $P^{\prime}$ and $P_{1}^{\prime}$ also satisfy (12), then

$$
P^{\prime} Q_{1}^{\prime}-P_{1}^{\prime} Q^{\prime}=R^{\prime}
$$

From (13) and (14),

$$
P=P^{\prime}+K Q^{\prime}, \quad K \text { a polynomial. }
$$

From (12), we see that

$$
\left|P-P^{\prime}\right|=|K|\left|Q^{\prime}\right|<|Q|=|D||Q|,
$$


which implies $|K|<|D|$. The number of possible polynomials $P$ satisfying (12) for a given $R$ is no greater than $q^{\operatorname{deg} D}$. (11) implies

$$
0 \neq|R|<\left|Q_{1}\right| \psi(Q)+|Q| \psi\left(Q_{1}\right)
$$

and we can only take polynomials $R$ divisible by $D$. We find that

$$
N\left(Q_{1}, Q\right) \leq \frac{\left|Q_{1}\right| \psi(Q)+|Q| \psi\left(Q_{1}\right)}{|D|}|D|=\left|Q_{1}\right| \psi(Q)+|Q| \psi\left(Q_{1}\right) .
$$

Then

$$
m\left(E_{Q_{1}} \cap E_{Q}\right) \leq 2 \psi\left(Q_{1}\right) \psi(Q) .
$$

Since $\sum_{\operatorname{deg} Q \leq n} \psi(Q)$ diverges,

$$
\sum_{\operatorname{deg} Q \leq n} \psi(Q) \leq\left(\sum_{\operatorname{deg} Q \leq n} \psi(Q)\right)^{2}
$$

for sufficiently large $n$. Therefore

$$
\begin{aligned}
\sum_{\operatorname{deg} Q_{1}, \operatorname{deg} Q \leq n} m\left(E_{Q_{1}} \cap E_{Q}\right) & \leq 2 \sum_{\substack{\operatorname{deg} Q_{1}, \operatorname{deg} Q \leq n \\
Q \neq Q_{1}}} \psi\left(Q_{1}\right) \psi(Q)+\sum_{\operatorname{deg} Q \leq n} \psi(Q) \\
& <3\left(\sum_{\operatorname{deg} Q \leq n} \psi(Q)\right)^{2}
\end{aligned}
$$

for all sufficiently large $\operatorname{deg} Q$. From (3) and (10), we have

$$
\sum_{\operatorname{deg} Q_{1}, \operatorname{deg} Q \leq n} m\left(E_{Q_{1}} \cap E_{Q}\right)<3 C^{2}\left(\sum_{\operatorname{deg} Q \leq n} m\left(E_{Q}\right)\right)^{2}
$$

for infinitely many $Q$. Hence $m(E)>\left(3 C^{2}\right)^{-1}$, by [9, Lemma 5, pp. 17-18]. Finally, applying Theorem 4, we have the assertion of the theorem.

Example 2. Put

Then

$$
\psi(Q)= \begin{cases}1 /|Q| & \text { if } Q \text { is irreducible } \\ 0 & \text { otherwise }\end{cases}
$$

$$
\sum_{n=1}^{\infty} \sum_{Q: \operatorname{deg} Q=n} \psi(Q)>\sum_{k=1}^{\infty} \frac{1}{q^{k}} \cdot \frac{1}{k} \cdot q^{k}=\infty
$$

and it is easy to see that

$$
\sum_{\operatorname{deg} Q \leq n} \psi(Q) \leq C \sum_{\operatorname{deg} Q \leq n} \psi(Q) \frac{\Phi(Q)}{|Q|} .
$$

Thus there are infinitely many solutions $P / Q$ of

$$
\left|f-\frac{P}{Q}\right|<\frac{1}{|Q|^{2}}, \quad Q \text { is irreducible, }
$$

for a.e. $f \in \mathbb{L}$. 


\section{References}

[1] V. Berthé and H. Nakada, On continued fraction expansions in positive characteristic: equivalence relations and some metric properties, Exposition. Math. 18 (2000), 257-284.

[2] P. Billingsley, Ergodic Theory and Information, Wiley, New York, 1965.

[3] E. Carlitz and E. Cohen, Divisor functions of polynomials in a Galois field, Duke Math. J. 14 (1947), 13-20.

[4] R. J. Duffin and A. C. Schaeffer, Khintchine's problem in metric diophantine approximation, ibid. 8 (1941), 243-255.

[5] M. Fuchs, On metric Diophantine approximation in the field of formal Laurent series, Finite Fields Appl. 8 (2002), 343-368.

[6] P. X. Gallagher, Approximation by reduced fractions, J. Math. Soc. Japan 13 (1961), 342-345.

[7] B. de Mathan, Approximations diophantiennes dans un corps local, Bull. Soc. Math. France Suppl. Mém. 21 (1970).

[8] W. Philipp, Some metrical theorems in number theory, Pacific J. Math. 20 (1967), 109-127.

[9] V. G. Sprindžuk, Metric Theory of Diophantine Approximations, Wiley, New York, 1979.

Department of Mathematics

Keio University

3-14-1 Hiyoshi, Kohoku-ku

Yokohama, 223-8522, Japan

E-mail: kae@math.keio.ac.jp

nakada@math.keio.ac.jp

Received on 5.2.2001

and in revised form on 22.10.2002 